\title{
Legionella pneumophila Serogroup 9
}

National Cancer Institute

\section{Source}

National Cancer Institute. Legionella pneumophila Serogroup 9. NCI Thesaurus. Code C123506.

Subgroup 9 of Legionella pneumophila, differentiated by its antigenicity. 